Micro-chaotic dynamics due to digital sampling in hybrid systems of Filippov type

Glendinning, $\mathrm{P}$ and Kowalczyk, $\mathrm{P}$

2010

MIMS EPrint: 2010.1

Manchester Institute for Mathematical Sciences

School of Mathematics

The University of Manchester

\footnotetext{
Reports available from: http://eprints.maths.manchester.ac.uk/

And by contacting: The MIMS Secretary

School of Mathematics

The University of Manchester

Manchester, M13 9PL, UK
} 


\title{
Micro-chaotic dynamics due to digital sampling in hybrid systems of Filippov type
}

\author{
P. Glendinning a and P. Kowalczyk ${ }^{\mathrm{b}, *}$ \\ ${ }^{a}$ Centre for Interdisciplinary Computational and Dynamical Analysis (CICADA) \\ and School of Mathematics, University of Manchester, Manchester, M13 9PL, \\ U.K. \\ ${ }^{\mathrm{b}}$ Centre for Interdisciplinary Computational and Dynamical Analysis (CICADA), \\ University of Manchester, Manchester, M13 9PL, U.K.
}

\begin{abstract}
This paper describes a novel mechanism leading to the sudden onset of chaos that may occur in Filippov type systems when the switching decision is made at discrete times. Such systems can be thought of as models of switched digital control systems. Our findings are explained in detail using a representative planar example. Results are then extended to more general planar switched systems with digital sampling. It is conjectured that this mechanism is also typical of $n$-dimensional switched systems with digital sampling, and numerical investigations of a third order relay feedback system are presented to support this; the onset of chaos is triggered in the way which is equivalent to that revealed for the planar case.
\end{abstract}

Key words: chaotic attractor, switched systems, digital sampling

\section{Introduction}

The use of a digital computer as a controller device grown in the past decades leading to a widespread application of digital control systems $[1,2]$. Nowadays digital control

* Corresponding author.

Email address: piotr.kowalczyk@manchester.ac.uk (P. Kowalczyk).

Preprint submitted to Elsevier

8 September 2009 
systems occur in a plethora of applications ranging from chemical processes, aircraft and traffic control to process control in industries such as machine tools production[35]. The control, design and analysis of these control systems involves understanding the interaction between continuous and discrete dynamics. For example, the automated control of a car moving on a road is implemented by digital computer but the motion of a car is continuous in time [6]. Computers that are used in such a system send a digital signal which is then converted to an analogue signal and can be fed into the actuator. The digital signal which corresponds to a finite sequence of numbers leaves or enters the computer at some time intervals, say $\tau$, which we will term a sampling time. In this paper we will consider $\tau$ as a constant, so the sampling times are at integer multiples of a fixed $\tau>0$, but in general $\tau$ could be a function of time. Since the digital signal has to be converted into a continuous time signal, and vice versa, the design of the control of such a system needs to take into account the effects of the interaction between the continuous and discrete dynamics.

These types of control systems are often referred to in the control literature as hybrid control systems [7]. In [3,4] it has been shown that the digitization of the spatial structure by the controller can induce micro-chaotic transient dynamics. Effects of digitization on the stability of the solutions have been considered in $[8,9]$, and in [10] the existence of different types of attractors in a simple model of a delta-modulated control system has been shown. Here we consider another aspect of digitization in hybrid systems. We assume that the input to the controller is delivered at discrete times, separated by a constant $\tau>0$, and show that for arbitrarily small $\tau$ the system can exhibit chaotic dynamics. Moreover, there are scaling laws relating the maximum distance of the chaotic attractor due to digital sampling from the simple periodic attractor of the continuously sampled system. It is linear for sufficiently small values of $\tau$ but at larger $\tau$ there is a change in the properties of the boundedness of the chaotic attractor and the scaling becomes nonlinear. Our findings will be illustrated and explained using general planar hybrid systems of Filippov type with digital sampling. That is we consider systems with discontinuous vector fields where the control input is digitized; it is available at discrete time intervals. We will then argue that our findings generalize to $n$-dimensional systems. This conjecture will be motivated by presenting numerical findings of a general third-order piecewise linear relay feedback system with digital sampling.

This paper is arranged as follows. In Sec. 2 planar Filippov type systems with digital sampling are introduced. In Sec. 3 the complex dynamics pertaining to these types of systems will be presented using a simple planar example. The observed dynamics will be explained in detail in the same section and the existence of an attractor with 
positive Lyapunov exponents for arbitrarily small $\tau$ will be proven, even though the sliding orbit for $\tau=0$ is stable. Theoretical arguments will be presented showing how these results extend to more general planar systems. This extension will be given in Sec. 4. There we will also show that Filippov's construction that determines the vector field that gives so-called sliding flow is a natural limit when the sampling time $\tau \rightarrow 0$ for a flow that gives zig-zag trajectory evolving along the surface where the switchings between different vector fields occur. This provides further justification for Filippov's construction of the sliding flow. In Sec. 5 we will show numerically that similar results hold for a third order relay feedback system. In particular, the sudden onset of chaos as a result of the introduction of digital sampling is observed. We will argue that the mechanism leading to the onset of chaos is the same as that described for the planar case. Therefore the analysis of the planar systems is relevant to $n$-dimensional systems of Filippov type with digital sampling.

\section{$2 \quad$ Filippov type systems with digital sampling}

We start our investigations by considering Filippov systems for which the evolution of a variable $x$ in some region $D \subseteq \mathbb{R}^{2}$ is determined by the equations

$$
\dot{x}(t)=\left\{\begin{array}{lll}
F_{1}(x(t), \mu) & \text { if } \quad H(x(t), \mu)>0 \\
F_{2}(x(t), \mu) & \text { if } \quad H(x(t), \mu)<0
\end{array}\right.
$$

where $F_{1}, F_{2}$ are sufficiently smooth vector functions, $F_{1}, F_{2}: \mathbb{R}^{2} \times \mathbb{R}^{m} \mapsto \mathbb{R}^{2}$ and $H(x(t), \mu): \mathbb{R}^{2} \times \mathbb{R}^{m} \mapsto \mathbb{R}$ is some smooth scalar function depending on the system states $x \in \mathbb{R}^{2}$, and parameter $\mu \in \mathbb{R}^{m} ; t \in \mathbb{R}$ is the time variable. We leave the description of the switching behaviour when $H(x, \mu)=0$ to a little later, above equation (3). Let us define the boundary $\Sigma$ as

$$
\Sigma:=\left\{x \in \mathbb{R}^{2}: H(x(t), \mu)=0\right\}
$$

which divides the region $D$ into two subspaces,

$$
G_{1}:=\left\{x \in \mathbb{R}^{2}: H(x, \mu)>0\right\},
$$

and

$$
G_{1}:=\left\{x \in \mathbb{R}^{2}: H(x, \mu)<0\right\},
$$

in which the dynamics is smooth. Depending on the direction of the vector fields with respect to $\Sigma$ those trajectories starting in $G_{1}$ and $G_{2}$ that reach $\Sigma$ in finite 
time will either cross it or evolve along $\Sigma$ (the so-called sliding motion). Let $\sigma(x)=$ $\left\langle H_{x}, F_{1}\right\rangle\left\langle H_{x}, F_{2}\right\rangle$, where $\langle$,$\rangle denotes the dot product and H_{x}$ the vector normal to $\Sigma$. The switching surface $\Sigma$ can be divided into subsets, say $\Sigma_{c}$ and $\hat{\Sigma}$ defined as

$$
\Sigma_{c}:=\{x \in \Sigma: \sigma(x)>0\}, \quad \hat{\Sigma}:=\{x \in \Sigma: \sigma(x) \leq 0\}
$$

Trajectories generated by $F_{1}$ or $F_{2}$ that reach $\Sigma_{c}$ switch between each other upon the evolution across $\Sigma_{c}$. Note that the trajectories so obtained are continuous, and are built of segments generated by $F_{1}$ and $F_{2}$. If on the other hand $\hat{\Sigma}$ is reached from $G_{1}$ or $G_{2}$, then the motion follows so called sliding flow along $\hat{\Sigma}$, and the vector field that generates this motion is defined as

$$
F_{s}=\alpha_{s} F_{1}+\left(1-\alpha_{s}\right) F_{2}
$$

where $\alpha_{s}=\alpha_{s}(x)=\frac{\left\langle H_{x}, F_{2}\right\rangle}{\left\langle H_{x},\left(F_{2}-F_{1}\right)\right\rangle}$, and $0 \leq \alpha_{s}(x) \leq 1$.

In (1) the control of the switching between the two systems across $\Sigma$ is instantaneous. The modified hybrid Filippov systems we study are obtained by assuming that the control function $H$ is evaluated at discrete times $k \tau, k=0,1,2, \ldots$, for some constant $\tau>0$, and so the decision to change evolution equation can only occur at these discrete times. Thus for $k=0,1,2,3, \ldots$ we define a discrete variable $i_{k}$ by

$$
i_{k+1}=\left\{\begin{array}{l}
1 \quad \text { if } \quad H(x(k \tau), \mu)>0 \\
2 \quad \text { if } \quad H(x(k \tau), \mu)<0 \\
i_{k} \quad \text { if } \quad H(x(k \tau), \mu)=0
\end{array}\right.
$$

with $i_{0}=1$ if $H(x(0), \mu)=0$ (arbitrarily chosen) so that $i_{1}$ is always well defined, and replace the evolution (1) by

$$
\dot{x}(t)=F_{i_{k}}(x(t), \mu) \quad \text { if } \quad(k-1) \tau \leq t<k \tau .
$$

Note that this system excludes the possibility of sliding motion, that is a motion within the discontinuity set $\Sigma$. 


\section{A simple example}

\subsection{Complex non-periodic motion}

To highlight the main features of our findings we will investigate in detail a simple planar example for which $F_{1}$ is based on the normal form of the subcritical Hopf bifurcation and $F_{2}$ is constant. Set

$$
F_{1}=\left(\begin{array}{c}
-\alpha x-\omega y+x\left(x^{2}+y^{2}\right) \\
\omega x-\alpha y+y\left(x^{2}+y^{2}\right)
\end{array}\right), \quad F_{2}=\left(\begin{array}{l}
a \\
b
\end{array}\right),
$$

$H(\mathbf{x})=x-\mu$, with $H$ given at times $k \tau(k=0,1,2, \cdots)$, where $\alpha, \omega, a, b, \mu$ and $\tau$ are some chosen constants (system parameters), and $\mathbf{x}=(x, y)^{T}$ is the state vector. We will note here that the sliding segment of the Filippov system (6) with no sampling applied terminates at the point $\mathbf{x}_{\mathbf{g}}=\left(\mu, y_{g}\right)$ which is where the solution of $F_{1}$ is tangential to the surface $\Sigma$ ( $y_{g}$ is close to 0 for $\alpha$ close to $\mu^{2}$ ). This implies that $\dot{x}=0$ at $\mathbf{x}_{\mathbf{g}}$.

The vector field $F_{1}$ is the normal form for a simple subcritical Hopf bifurcation, with a stable focus at the origin and an unstable periodic orbit with radius $\sqrt{\alpha}$ if $\alpha>0$. For appropriate choices of $\mu, a$ and $b$ the vector field $F_{2}$ can be used to create a stable periodic orbit for the Filippov system (1) as shown in Figure 1(a). The stability is derived from the fact that part of the cycle lies on $\Sigma$, and this segment of the orbit is called a sliding segment [11]. This stable cycle may coexist with the unstable cycle of the vector field $F_{1}$.

Let $x=\rho \cos \theta$ and $y=\rho \sin \theta$. We can then express vector field $F_{1}$ in polar form as

$$
F_{1}^{P}=\left(\begin{array}{c}
\rho\left(\rho^{2}-\alpha\right) \\
\omega
\end{array}\right)
$$

Thus, we can obtain the closed form solution for the flow generated by $F_{1}^{P}$, say $\phi_{1}^{P}=\phi_{1}^{P}(\rho, \theta)$. This closed form solution is given by

$$
\rho(t)=\sqrt{\frac{\alpha}{1-\left(1-\alpha \rho_{0}^{-2}\right) \exp 2 \alpha t}}, \quad \theta(t)=\theta_{0}+\omega t,
$$




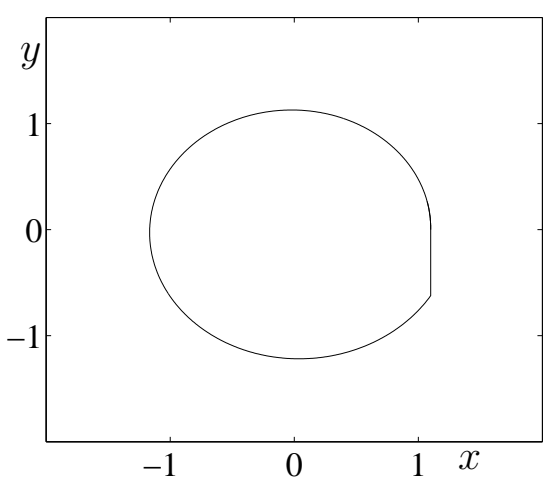

(a)

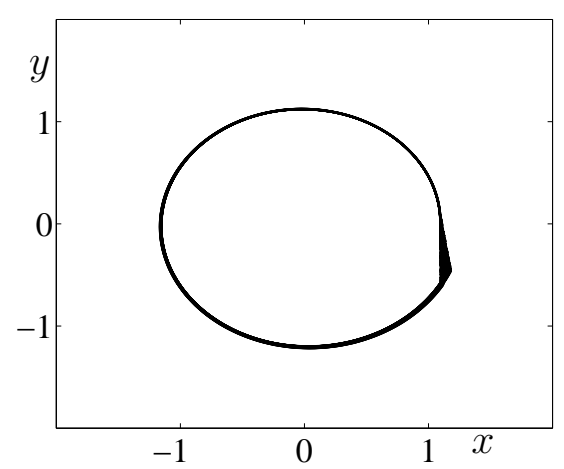

(b)

Fig. 1. Asymptotic trajectories in Filippov system (6) without digital sampling (a), and with digital sampling $\tau=0.01$ (b). Other parameters are set to $\omega=15, \alpha=1, \mu=1.1$.

where $\left(\rho_{0}, \theta_{0}\right)$ are the initial conditions. Note that for $\alpha>0$ there is a finite time blow-up of the system, hence $\omega$ must be sufficiently large so that the system switches before $\rho$ diverges.

For all the following numerical computations we set $\omega=15, \alpha=1$. In this case the vector field unstable limit cycle of $F_{1}$ is centered at the origin and has radius 1. The vector field $F_{2}$ is assumed constant; we set $a=-1$ and $b=1$. Finally let us set $\mu=1.1$ so that $\Sigma$ does not intersect the unstable limit cycle of $F_{1}$. For these parameters (1) with vector fields given by (6) admits two limit cycles: the stable limit cycle with sliding already referred to and the unstable cycle of $F_{1}$.

Let us now increase the sampling time $\tau$ from 0 and investigate the dynamics of (6) further. As Fig. 1(b) shows, there is an apparent thickening of the attractor: the stable limit cycle no longer exists and we observe an onset of more complex asymptotic dynamics. It turns out that this complex dynamics is chaotic and so there is a transition from a stable orbit to a chaotic attractor due to an introduction of the sampling process. This will be proven in the theoretical part of this section.

Let us determine the size of the attractor, measured as the distance from the largest to smallest values which the attractor attains on a Poincaré section defined on a set $\{y=0,-1.3<x<-1\}$.

\subsection{Scaling}

In Fig. 2 using the logarithmic scales we are depicting how the size of the attractor scales against the sampling time $\tau$. The dashed diagonal line refers to the linear 


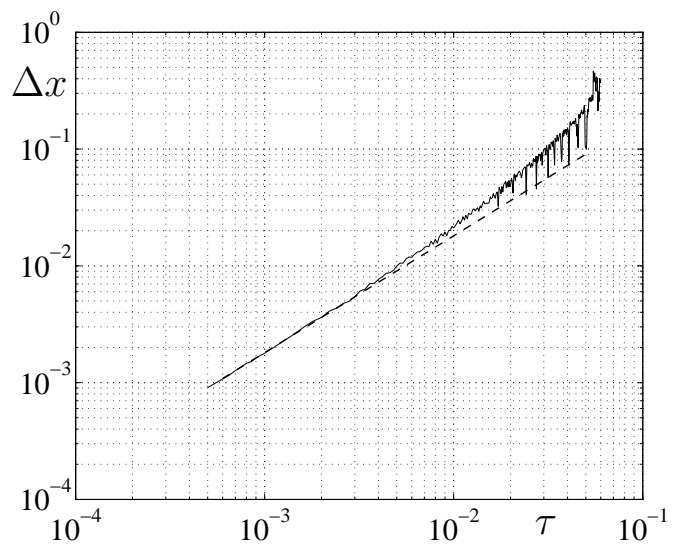

Fig. 2. Size of the attractor versus the sampling time $\tau$. The dashed line refers to theoretical predictions of the size of the attractor.

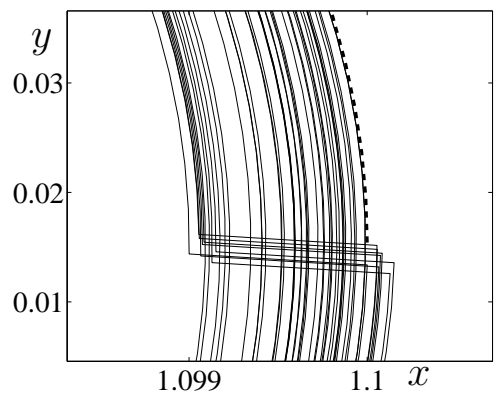

(a)

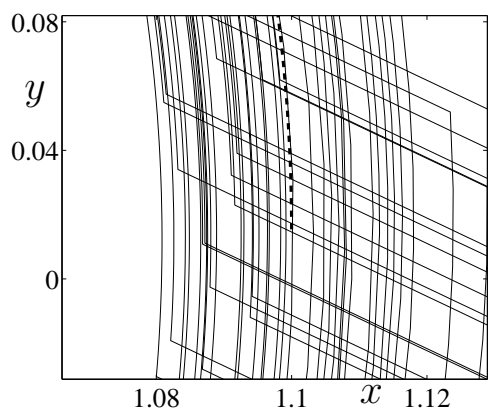

(b)

Fig. 3. Zoom into the region where the flow $\phi_{1}$ leaves $\Sigma$ tangentially, (a) for $\tau=0.001$ and (b) for $\tau=0.02$. Other parameters are set to $\omega=15, \alpha=1, \mu=1.1$.

scaling proportional to $\tau$ times a coefficient. We can see that for small values of $\tau$ the size of the attractor is proportional to $\tau$ but the increase in the value of the sampling time $\tau$ above $\tau=0.01$ results in the growing discrepancy between the numerical curve and the linear scaling. As it will be shown later for small values of $\tau$ the attractor is bounded by the trajectory leaving $\Sigma$ at $\mathbf{x}_{\mathbf{g}}$ which is not the case for larger values of $\tau$. This implies further that if we choose for a Poincaré section on which we measure the width of the attractor a line segment that crosses $\Sigma$ at $\mathbf{x}_{\mathbf{g}}$ along the $x$-coordinate then the scaling should be directly proportional to $\tau$ for sufficiently small values of $\tau$ i.e. the coefficient of proportionality would be 1 .

However, the size of the attractor is measured on section $\{y=0,-1.3<x<-1\}$. 
Therefore, we have to determine the expansion of the attractor along the flow after time $\pi / \omega$ which is the time required to map points from the neighborhood of point $\mathbf{x}_{\mathbf{g}}$ onto our chosen Poincaré section. This expansion is captured by the non-trivial Floquet multiplier of the fundamental solution matrix[12]. To find this multiplier we use the explicit solutions of the differential equations that define $F_{1}^{P}$, namely we use equation (7). After differentiating $\rho(t)$ with respect to $\rho_{0}$ we get

$$
\frac{d \rho}{d \rho_{0}}=\frac{\exp (2 t) \rho_{0}}{\left(\sqrt{-\rho_{0}^{2} /\left(\exp (2 t) \rho_{0}^{2}-\exp (2 t)-\rho_{0}^{2}\right)}\left(\exp (2 t) \rho_{0}^{2}-\exp (2 t)-\rho_{0}^{2}\right)^{2}\right)} .
$$

We then substitute our numerical values of $t$ and $\rho_{0}$ into (8). Namely we set for $t=\pi / \omega=\pi / 15$, and for $\rho_{0}=1.1$. We find that $\frac{d \rho}{d \rho_{0}}=1.8084$.

We should remark here that the choice of the Poincaré section along the $x$-axis implies that the expansion along the radius $\rho$ corresponds to the expansion along the $x$-axis and we do not need to consider any corrections implied by the fact that we obtained our multiplier using polar coordinates. However we should note that for our numerical values the $y$ component of $\mathbf{x}_{\mathrm{g}}$ is $y_{g} \approx 0.0154$ which implies that the angular distance from the $x$-axis to reach $x=y_{g}$-axis is $\arctan (0.0154 / 1.1) \approx 0.014 \ll \pi / \omega \approx 0.21$. Therefore, the error in $\frac{d \rho}{d \rho_{0}}$ implied by the fact that we have to measure the expansion from the $x$-axis back to the $x$-axis so that $\frac{d \rho}{d \rho_{0}}=\frac{d x}{d x_{0}}$ is negligible. The dashed line in Fig. 2 that corresponds to linear scaling has been obtained using this prediction. As we mentioned before there is a good agreement between this graph and the numerical scaling for small values of $\tau(\tau<0.01)$.

The numerical graph captures other effects such as resonances between the sampling time $\tau$ and the rotation $\omega$ of the flow $\phi_{1}$ which produce local variations in the scaling law visible in Fig. 2.

Before explaining where does the linear scaling come from and when does it fail let us focus on Fig. 3. In Fig. 3(a) we zoom into the region in the neighborhood of point $\mathbf{x}_{\mathbf{g}}$ for $\tau=0.001$. The dashed line in the figure denotes the trajectory generated by $F_{1}$ that starts at $\mathbf{x}_{\mathbf{g}}$. Apparently this trajectory bounds the attractor to the right. Moreover we note that furthest to the left where a trajectory generated by $F_{2}$ can reach along the $x$-coordinate on the level line $y=y_{g}$ is equal to $\mu-\tau$ which in our case is 1.099, and this is markedly visible in the figure. Increasing the value of $\tau$ leads to the case that the trajectory starting at $\mathbf{x}_{\mathbf{g}}$ no longer bounds the attractor to the right as it is visible in Fig. 3(b) (see the dashed trajectory). This suggests that it is 


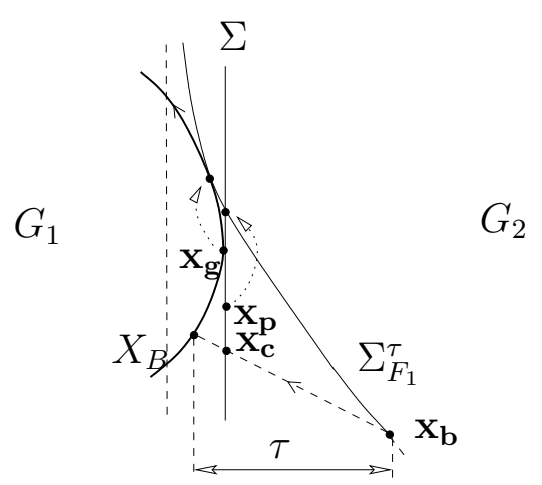

Fig. 4. Schematic representation of the bounding region $X_{B}$ for small values of the sampling time $\tau$.

the bounding of the attractor to the right by the trajectory starting at $\mathbf{x}_{\mathbf{g}}$ that allows for the linear scaling and when this fails also the linear scaling fails. Note that the left bound $\mu-\tau$ is directly proportional to $\tau$. To see if this could be the mechanism behind the observed scaling we consider a sufficiently small $\tau$. Define $\Sigma_{F_{1}}^{\tau}$ as the image of $\Sigma$ under the action of $\phi_{1}$ for time $\tau$. Let $\mathbf{x}_{\mathbf{p}} \in \Sigma$ be the pre-image of the point at which $\Sigma_{F_{1}}^{\tau}$ crosses $\Sigma$ in the neighborhood of $\mathbf{x}_{\mathbf{g}}$ as shown in Fig. 4. Define $X_{B}$ to be the set of initial points $\mathbf{x}$ in the neighborhood of $\mathbf{x}_{\mathbf{g}}$ such that for any $\mathbf{x} \in X_{B}$ a trajectory generated by $F_{1}$ evolves through $\mathbf{x}_{\mathrm{g}}$.

For $\tau$ sufficiently small $\Sigma_{F_{1}}^{\tau}$ is nearly tangent to $\Sigma$ and there exists a point on $\Sigma_{F_{1}}^{\tau}$, say $\mathbf{x}_{\mathbf{b}}$, such that the trajectory starting at $\mathbf{x}_{\mathbf{b}}$ crosses $\Sigma$ at some point $\mathbf{x}_{\mathbf{c}}$ below $\mathbf{x}_{\mathbf{p}}$, and the time of evolution from $\mathbf{x}_{\mathbf{b}} \in \Sigma_{F_{1}}^{\tau}$ to $X_{B}$ is $\tau$ (see Fig. 4). Therefore a trajectory starting at $\mathbf{x}_{\mathbf{b}}$ must switch to $\phi_{1}$ on $X_{B}$. It then follows that all trajectories rooted in $G_{1}$ below the line joining $\mathbf{x}_{\mathbf{b}}$ to $X_{b}$, after a finite number of switchings between flow $\phi_{1}$ and $\phi_{2}$ must reach the region bounded by $\Sigma, \Sigma_{F_{1}}^{\tau}$, and the line segment joining $\mathbf{x}_{\mathbf{b}}$ with $X_{B}$.

Note that since $\mathbf{x}_{\mathbf{p}}$ lies above $\mathbf{x}_{\mathbf{c}}$ no trajectory generated solely by $F_{1}$ can lie to the right of $X_{B}$ - penetrate $G_{2}$ and return to $G_{1}$ without switching to $\phi_{2}$. Finally any trajectory rooted at any point within the region bounded by $\Sigma, \Sigma_{F_{1}}^{\tau}$, and the line segment joining $\mathbf{x}_{\mathbf{b}}$ with $X_{B}$ switches to the vector field $F_{1}$ in a region to the left of $X_{B}$ - the time of evolution from any point in this region to reach some point in $G_{1}$ to the left of $X_{B}$ is less than $\tau$. Hence the trajectories that reach this region must leave it without being able to cross $\Sigma_{F_{1}}^{\tau}$. It then follows that for sufficiently small $\tau, X_{B}$ is a bounding set for the attractor. To find the bounding set to the left of $\Sigma$ we note that the set of points furthest to the left of $\Sigma$, which can be reached by a trajectory generated by $F_{2}$, has co-ordinates $(\mu-\tau, y)$. This implies that along the 
$x$ co-ordinate, in the neighborhood of $\mathbf{x}_{\mathbf{g}}$, the difference between the largest and the smallest values of $x$ on the attractor is $\tau$.

For larger values of the sampling time $\tau$ point $\mathbf{x}_{\mathbf{p}}$ might no longer lie above $\mathbf{x}_{\mathbf{c}}$, and there exist trajectories in the neighborhood of $\mathbf{x}_{\mathbf{g}}$ solely generated by $F_{1}$ that lie to the right of $X_{B}$. In this case the difference between the largest and the smallest values of $x$ on the attractor is different from $\tau$. Boundedness in the latter case will be proven in the next section.

\subsection{Boundedness}

We will prove this in two parts. First we show that if $\tau>0$ is sufficiently small then there is a compact set which solutions cannot leave, and hence which contains at least one attractor. This part of the proof is based on showing monotonic crossing of the local transversal by a trajectory, similarly as in the Poincaré- Bendixson Theorem [13]. We present this argument in fairly general terms below so that the extension to similar systems is clear. Second we show that any solution in this compact set (and hence the attractor itself) has a positive Lyapunov exponent.

The compact invariant region is annular, and its inner boundary is the unstable cycle of $F_{1}$ with radius one. To begin the construction of the outer boundary, note that the sliding segment of the (true) Filippov system terminates at the point $\mathbf{x}_{\mathbf{g}}=\left(\mu, y_{g}\right)$. Now, let $\phi_{1}^{t}(u, v)$ denote the flow generated by $F_{1}$, i.e. solutions of $F_{1}$ at time $t$ with initial conditions $(u, v)$. Define $V$ to be the set of points $(x, y)$ which can be reached from a point $(u, v) \in G_{1} \cup \Sigma$, with $v \geq-R$ within time $\tau$, and whose trajectory intersects $G_{2}$ in time $\tau$, namely

$$
\begin{aligned}
V=\left\{\left(x_{1}, x_{2}\right) \mid\left(x_{1}, x_{2}\right) \in \phi_{1}^{\tau}(u, v)\right. & \text { for some }(u, v) \in G_{1} \cup \Sigma \\
& \text { with } \left.v \geq-R \text { and } \phi_{1}^{\tau}(u, v) \cap G_{2} \neq \emptyset\right\}
\end{aligned}
$$

where $\phi_{i}^{t}(u, v)$ is the solution at time $t$ of the differential equation defined by $F_{i}$, $i=1,2$, with initial condition $(u, v)$.

Finally, let $V_{R}$ be the right hand boundary of $V$, i.e. $(u, v) \in V_{R}$ such that if $\left(u^{\prime}, v\right) \in V$ then $u \geq u^{\prime} . V_{R}$ is the time $\tau$ image of points on $\Sigma$ but close to $\mathbf{x}_{\mathbf{g}}$ this might not be the case. $V_{R}$ therefore represents a boundary which no orbit which starts in $G_{1}$ above $-R$ can cross within time $\tau$ under the flow $\phi_{1}$. The choice of $R$ is determined by later 
(a)

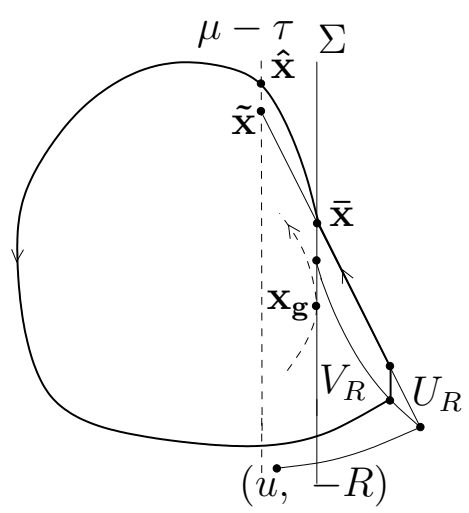

(b)

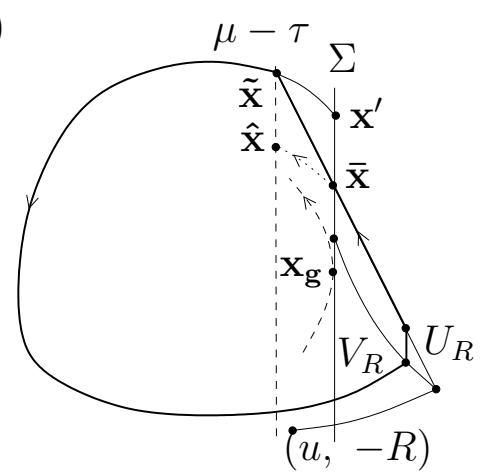

Fig. 5. Schematic representation of the bounding regions along $\Sigma$ and the boundaries $U_{R}$ and $V_{R}$ in the case (a) when $\hat{y}>\tilde{y}$, and (b) $\hat{y}<\tilde{y}$.

considerations, it needs to be large enough to allow the argument to close up below - numerical experiments show that $R=2$ is sufficiently large here.

Now consider the effect of the flow generated by $F_{2}$ to points in $G_{2}$ to the left of $V_{R}$. The trajectories are straight lines with slope -1 , and as $\dot{y}=-1$ the furthest to the left that an orbit from $G_{2}$ can reach in time $\tau$ has $x=\mu-\tau$. Let $U$ be the union of $V_{R}$ and the set of points on straight lines of slope -1 from $(u, v) \in V_{R}$ with $u>\mu-\tau$ to $\mu-\tau$. Finally let $U_{R}$ be the right hand boundary of $U$. Note that $U_{R}$ must be connected.

To summarise: by construction, in time $\tau$, no solution of $F_{1}$ above the line $y=-R$ can move to the right of $V_{R}$ (which is on the left of $U_{R}$ or equal to it at places), and under $F_{2}$ all such orbits remain to the left of $U_{R}$ until they return to $G_{1}$.

Let $\bar{y}$ be the highest point in $U_{R}$ with $\bar{x}=\mu$ and $\tilde{y}$ the highest point in $U_{R}$ with $\tilde{x}=\mu-\tau$. Let $\hat{\mathbf{x}}$ be the first intersection of the solution of $F_{1}$ through $\overline{\mathbf{x}}$ with $\hat{x}=\mu-\tau$.

If $\hat{y}>\tilde{y}$ then the outer boundary of the bounding region is the trajectory through $\hat{\mathbf{x}}$ under $F_{1}$ until it hits $V_{R}$ for the first time (see Figure 5(a)), a vertical line segment from $V_{R}$ to $U_{R}$, and then $U_{R}$ back to $\overline{\mathbf{x}}$. Note that this requires $R$ to be large enough so that the trajectory does hit $V_{R}$. If not then a larger $R$ needs to be chosen.

If $\hat{y}<\tilde{y}$ let $\mathbf{x}^{\prime}$ be the first preimage of $\tilde{\mathbf{x}}$ on $\Sigma$ under $F_{1}$, and note that $y^{\prime}$ will lie above $\bar{y}$. Then the outer boundary of the bounding region is the trajectory through $\mathbf{x}^{\prime}$ under $F_{1}$ until it hits $V_{R}$ for the first time, a vertical line segment from $V_{R}$ to $U_{R}$, and then $U_{R}$ back to $\tilde{\mathbf{x}}$ (see Figure $5(\mathrm{~b})$ ). As before $R$ needs to be large enough for 
the connections to work.

In either case we will have created a compact region which no trajectory can exit from, and hence the annular region contains at least one attractor. Note that in the current case we are not able to predict how the size of the attractor scales with respect to the sampling time $\tau$.

\subsection{Positive Lyapunov exponent}

In the following section we will show that, in the situation when the trajectories around the sliding cycle (the sliding cycle existing for $\tau=0$ ) are bounded for $\tau>0$, the Lyapunov exponent along the flow is positive. Let us assume $\alpha>0$. We then consider trajectories evolving in a bounded region, say $D$, where $D$ is a set of points in some sufficiently small neighbourhood of $\mathbf{x}_{\mathbf{g}}$. We define $D$ as an interior of a region bounded by $\{x-\mu+\tau=0\}$, and $\left\{(x, y) \in \mathbb{R}^{2}, t \in\left(-t_{1}, t_{2}\right): \phi_{1}\left(\mathbf{x}_{\mathbf{g}}, t\right)\right\}$, where $t_{i}$ $(i=1,2)$ are positive and are the times required to reach $\{x-\mu+\tau=0\}$ from $\mathbf{x}_{\mathbf{g}}$ in forward and backward time by following flow $\phi_{1}$, which is the flow generated by $F_{1}$, see Fig. 6. Note that $D$ is the region that must be visited by the chaotic trajectory every time it leaves the zig-zag part of the motion along $\Sigma$ when $\tau$ is sufficiently small, as we explained in Sec. 3.2. Each return of the trajectory from $D$ back to $D$ is made up of a section from $\left(x_{0}, y_{0}\right)$ at lift-off with $x<\mu$ back to $\left(x_{1}, y_{1}\right)$ at $x=\mu$ and on for time $t \in(0, \tau]$ to $\left(x_{2}, y_{2}\right)$ with $x_{2}>\mu$, then a zig-zag of sections each generated for a length of time $\mathcal{O}(\tau)$, with points with even indices to the right of $\Sigma$ and odd indices on the left, until we get back on the left for a further lift-off at $\left(x_{0}^{\prime}, y_{0}^{\prime}\right)$. The time to get from any point in $D$ to the first intersection with $\Sigma$ at $\left(x_{1}, y_{1}\right)$ is $t_{0} \approx \mathcal{O}(\pi / \omega)\left(t_{0}\right.$ is large comparatively to the time spent by the flow to follow each zig-zag segment). Let us suppose that the number of switchings between $\phi_{1}$ and $\phi_{2}$, and vice versa, to reach $\left(x_{0}^{\prime}, y_{0}^{\prime}\right)$ from $\left(x_{0}, y_{0}\right)$ is an odd number.

We wish to determine the average behavior of state vectors from region $D$ in the tangent space of the phase space, under the action of (6). This can be done by obtaining the linearized map that maps all points in $D$ back to itself. The logarithms of the eigenvalues of the linear map so obtained correspond to Lyapunov exponents. We will show that one of these Lyapunov exponents is positive.

To obtain the linearized map we can solve for the flow $\phi_{1}^{P}$ (solution of $F_{1}$ given in polar co-ordinates) and expand the solution for $\rho(t)$ about $\rho_{0}$ where $\rho_{0}$ is the initial radius from the origin to some point in $D$. We should note here that we consider cartesian 


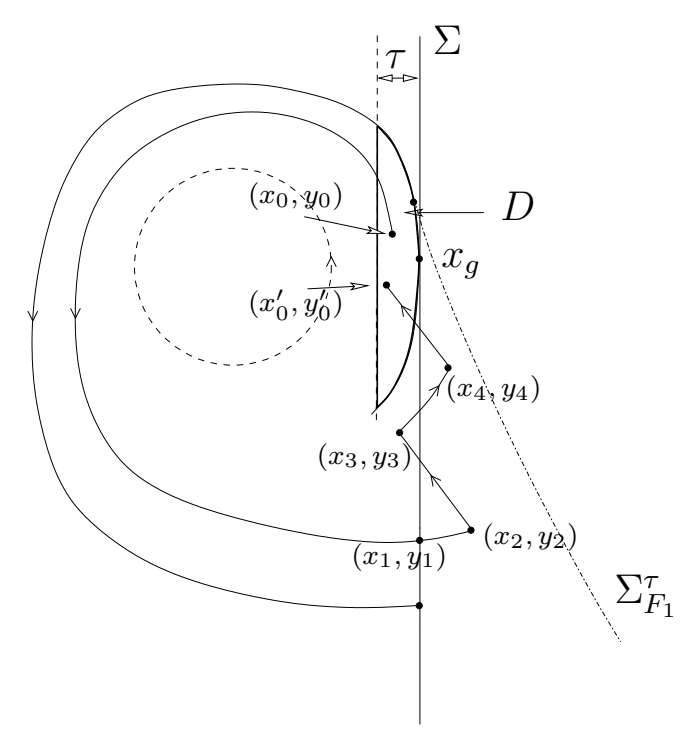

Fig. 6. Schematic representation of the segments of trajectories which built a map that maps region $D$ onto itself

and polar coordinate sets. Therefore we need to consider how vectors $\left(x^{T}, y^{T}\right)$ which live in the tangent space of vectors $(x, y)$ transform onto vectors $\left(\rho^{T}, \theta^{T}\right)$ which live in the tangent space of vectors $(\rho, \theta)$. Namely

$$
\begin{gathered}
\left(\begin{array}{l}
x^{T} \\
y^{T}
\end{array}\right)=\left(\begin{array}{cc}
\cos \theta & -\rho \sin \theta \\
\sin \theta & \rho \cos \theta
\end{array}\right)\left(\begin{array}{l}
\rho^{T} \\
\theta^{T}
\end{array}\right), \\
\left(\begin{array}{c}
\rho^{T} \\
\theta^{T}
\end{array}\right)=\left(\begin{array}{ll}
\frac{x}{\sqrt{x^{2}+y^{2}}} & \frac{y}{\sqrt{x^{2}+y^{2}}} \\
-\frac{y}{x^{2}+y^{2}} & \frac{x}{x^{2}+y^{2}}
\end{array}\right)\left(\begin{array}{l}
x^{T} \\
y^{T}
\end{array}\right) .
\end{gathered}
$$

Let $P C$ be the transformation matrix of (10) and $C P$ the transformation matrix of (11). Namely

$$
P C=\left(\begin{array}{cc}
\cos \theta & -\rho \sin \theta \\
\sin \theta & \rho \cos \theta
\end{array}\right), \quad C P=\left(\begin{array}{cc}
\frac{x}{\sqrt{x^{2}+y^{2}}} & \frac{y}{\sqrt{x^{2}+y^{2}}} \\
-\frac{y}{x^{2}+y^{2}} & \frac{x}{x^{2}+y^{2}}
\end{array}\right)
$$

Therefore, as we consider map $D \mapsto D$, and $D$ is given in the cartesian setting, we first have to use (11) to account for the transformation of the tangent space at $\left(x_{0}, y_{0}\right)$ to the corresponding tangent space at $\left(\rho_{0}, \theta_{0}\right)$. We then consider a map 
$\left(\rho_{0}, \theta_{0}\right) \mapsto\left(\rho_{1}, \theta_{1}\right)$ which linearization is given by the linear term of the expansions for $\rho(t)$ and $\theta(t)$ about $\left(\rho_{0}, \theta_{0}\right)$ for the initial segment of the trajectory. Using (7) we can show that the $\rho$ component of this linearization is given by

$$
f_{0}\left(t_{0}, \rho_{0}\right) \exp \left(2 \alpha t_{0}\right)
$$

with $f_{0}\left(0, \rho_{0}\right)=1 ; f_{0}$ is monotonically increasing for increasing time $t_{0}$ and it is given by

$$
f_{o}=\frac{\alpha^{2}}{\sqrt{\alpha\left(1-\left(1-\alpha \rho_{0}^{-2}\right) \exp \left(2 \alpha t_{0}\right)\right)^{-1}}\left(1-\left(1-\alpha \rho_{0}^{-2}\right) \exp \left(2 \alpha t_{0}\right)\right)^{2} \rho_{0}^{3}} .
$$

Similarly we can obtain the linearized coefficient for the expansion of $\theta(t)$ about the initial angle $\theta_{0}$. This coefficient is 1 . Since in polar coordinates both state variables are decoupled we can now write the transformation matrix for the linearized map $\left(\rho_{0}, \theta_{0}\right) \mapsto\left(\rho_{1}, \theta_{1}\right)$, which is equivalent to finding the monodromy matrix, say $M_{0}$, corresponding to the action of flow $\phi_{1}^{P}$ for time $t_{0}$. It has the diagonal form, and it is given by

$$
M_{0}=\left(\begin{array}{cc}
f_{0}\left(t_{0}, \rho_{0}\right) \exp \left(2 \alpha t_{0}\right) & 0 \\
0 & 1
\end{array}\right) .
$$

In the next step we map $\left(\rho_{1}, \theta_{1}\right) \mapsto\left(\rho_{2}, \theta_{2}\right)$. Since we follow $\phi_{1}^{P}$ the new monodromy matrix, say $M_{1}$, is given by

$$
M_{1}=\left(\begin{array}{cc}
f_{1}\left(t_{1}, \rho_{1}\right) \exp \left(2 \alpha t_{1}\right) & 0 \\
0 & 1
\end{array}\right) .
$$

Similarly as for $f_{0}, f_{1}\left(0, \rho_{1}\right)=1$ and $f_{1}$ is monotonically increasing in $t_{1}$. The functional expression for $f_{1}$ is the same as for $f_{0}$ (see equation (13)) with $\rho_{0}$ replaced by $\rho_{1}$, and $t_{0}$ replaced by $t_{1}$.

At this point, that is at $\left(\rho_{2}, \theta_{2}\right)$ a switching to $F_{2}$ occurs. Hence we have to obtain the monodromy matrix for the flow generated by vector field $F_{2}$. Considering the cartesian coordinate set $\left(x_{2}, y_{2}\right) \mapsto\left(x_{3}, y_{3}\right)$ is pure translation. Thus, the identity matrix corresponds to the linear part of the map. In other words the monodromy matrix corresponding to flow $\phi_{2}$ is given by

$$
M_{F_{2}}=\left(\begin{array}{ll}
1 & 0 \\
0 & 1
\end{array}\right) \text {. }
$$


Note, however, that $M_{F_{2}}$ corresponds to the monodromy matrix of $\phi_{2}$ in the cartesian coordinates. Thus we have to map $\left(\rho_{2}, \theta_{2}\right) \mapsto\left(x_{2}, y_{2}\right)$ to be able to use $M_{F_{2}}$. This transformation is given by (10). After the application of the translation map $\left(x_{2}, y_{2}\right) \mapsto\left(x_{3}, y_{3}\right)$ we have to return to polar coordinates by means of transformation (11). To obtain the final map we have to proceed with applications of linear transformation corresponding to the evolution using flow $\phi_{1}^{P}$, then apply transformation (10) following by the translation (which corresponds to the action of $\phi_{2}$ for some small time $\mathcal{O}\left(m_{i} \tau\right)$ where $m_{i}$ is a positive integer depending on the segment index $\left.i\right)$, and then again apply transformation (11). This sequence has to be repeated until we get mapped onto $D$.

To show that the linearized map is indeed characterized by a positive Lyapunov exponent what we need is the determinant of the product of $M_{i}(i=0,1,2,3, \cdots)$ and of the transformation matrices $C P$ and $P C$. Let us call this matrix product by $M$.

Consider now the segment from $\left(x_{0}, y_{0}\right)$ to $\left(x_{2}, y_{2}\right)$. Let $M_{01}$ be the monodromy matrix referring to this segment. Then $\operatorname{det}\left(M_{01}\right)$ is given by the product of the determinant of (11) which is $1 / \rho_{0}$, multiplied by (13) and $\rho_{2}$, where $\rho_{2}$ is given by (7). Taking this product and simplifying the resulting expression gives

$$
\operatorname{det}\left(M_{01}\right)=\frac{\alpha^{2} \exp (2 \alpha t)}{\left(\rho_{0}^{2}-\exp (2 \alpha t)\left(\rho_{0}^{2}-\alpha\right)\right)^{2}}
$$

where $t=t_{0}+t_{1}$. Note that $M_{01}$ is a matrix product of $P C M_{1} M_{O} C P$. To obtain the determinant of the full matrix $M$ we calculate the products of $M_{i}$ (that correspond to segments generated by $\left.\phi_{1}^{P}\right)$ together with the transformation matrices. Assume that the number of segments generated by $F_{1}$ to map $\left(x_{0}, y_{0}\right) \mapsto\left(x_{0}^{\prime}, y_{0}^{\prime}\right)$ is $N_{1}$. Then we use (14) with $\rho_{i}$ and $t_{i}$ for $i=3,5,7 \cdots 2 N_{1}-1$ for the remaining segments generated by $\phi_{1}^{P}$. Thus

$$
\operatorname{det}(M)=\frac{\alpha^{2} \exp \left(2 \alpha t_{0}+t_{1}\right)}{\left(\rho_{0}^{2}-\exp \left(2 \alpha t_{0}+t_{1}\right)\left(\rho_{0}^{2}-\alpha\right)\right)^{2}} \Pi_{i=2 k+1}^{2 N_{1}-1} \frac{\alpha^{2} \exp \left(2 \alpha t_{i}\right)}{\left(\rho_{i}^{2}-\exp \left(2 \alpha t_{i}\right)\left(\rho_{i}^{2}-\alpha\right)\right)^{2}},
$$

for $k=1,2,3 \cdots$.

We now need to determine if $\operatorname{det}(M)>1$. We can write

$$
\frac{\alpha^{2}}{\left(\rho_{0}^{2}-\exp \left(2 \alpha t_{0}+t_{1}\right)\left(\rho_{0}^{2}-\alpha\right)\right)^{2}}=\frac{1}{\left(\rho_{0}^{2} / \alpha-\exp \left(2 \alpha t_{0}+t_{1}\right)\left(\rho_{0}^{2} / \alpha-1\right)\right)^{2}} \geq 1 .
$$

Note that if $t_{0}+t_{1}=0$ the above coefficient is 1 and for increasing times it is 
monotonically increasing. Since we assumed $\omega$ large enough and hence $t_{0}+t_{1}$ must be such that this coefficient is bounded. Similarly

$$
\frac{\alpha^{2}}{\left(\rho_{i}^{2}-\exp \left(2 \alpha t_{i}\right)\left(\rho_{i}^{2}-\alpha\right)\right)^{2}}=\frac{1}{\left(\rho_{i}^{2} / \alpha-\exp \left(2 \alpha t_{i}\right)\left(\rho_{i}^{2} / \alpha-1\right)\right)^{2}} \geq 1
$$

for $i=3,5,7 \cdots 2 N_{1}-1$. Therefore $\operatorname{det}(M)>1$ since $t_{0}+t_{1}$ and $t_{i}$ (for $i=$ $\left.3,5,7 \cdots 2 N_{1}-1\right)$ are positive. Now, let $\lambda_{1}$, and $\lambda_{2}$ be the eigenvalues of $M$. Then, $\operatorname{det}(M)=\lambda_{1} \lambda_{2}>1$, and both $\lambda_{1}, \lambda_{2}$ must be either positive or negative, and the modulus of at least one of the eigenvalues must be greater than 1 . Let $\lambda_{1}$ be the eigenvalue with the larger modulus. Then the Lyapunov exponent that corresponds to this eigenvalue is given by

$$
\lambda_{1}^{E x p}=\frac{1}{t_{0}+\sum_{i=2 k-1}^{2 N_{1}-1} t_{i}+\bar{t}} \ln \left|\lambda_{1}\right|>0,
$$

for $k=1,2,3, \cdots$ where $\bar{t}$ is the total time of evolution following $\phi_{2}$ and $\bar{t} \approx \mathcal{O}(\tilde{m} \tau)$ where $\tilde{m}$ is a positive integer corresponding to the total number of segments generated by $F_{2}$ along the zig-zag motion.

\section{Remark}

We should remark here that to obtain the Lyapunov exponents we should evaluate the flow for the time $t \rightarrow \infty$. However, in the current case, for sufficiently small $\tau$ the variations of the affine map $D \mapsto D$, and hence the variations of monodromy matrix $M$ can be considered negligible for all points in $D$.

\subsection{Alternative calculation of the determinant}

From standard theory the determinant of a monodromy matrix, say $\Phi$, along the flow $f$ is given by

$$
\operatorname{det}(\Phi)=\exp \left(\int_{0}^{t} \nabla \cdot f d s\right)
$$

Of course, the divergence of the constant vector field is zero, so in our case the only contribution is from the vector field to the left of $\Sigma$. Applying the nabla operator to $F_{1}$ given by (6) yields

$$
\nabla \cdot f=-2 \alpha+4\left(x^{2}+y^{2}\right)=-2 \alpha+4 \rho^{2}
$$


where $\rho=\sqrt{x^{2}+y^{2}}$. Indeed, as we have mentioned before, in polar coordinates (6) becomes

$$
\begin{aligned}
& \dot{\rho}=-\alpha \rho+\rho^{3} \\
& \dot{\theta}=\omega
\end{aligned}
$$

These equations are easily integrated with initial conditions $\left(\rho_{0}, \theta_{0}\right): \theta=\theta_{0}+\omega t$ and

$$
\rho^{2}=\frac{\alpha}{1-A e^{2 \alpha t}}=\alpha\left(1+\frac{A \exp (2 \alpha t)}{1-A \exp (2 a t)}\right)
$$

for $\rho_{0}^{2}>\alpha$ with $A=\frac{\rho_{0}^{2}-\alpha}{\rho_{0}^{2}}<1$. From (19) and (17), whilst $\rho$ remains finite and to the left of $\Sigma$

$$
\int_{0}^{t} \nabla \cdot f d s=2(\alpha t-\ln (1-A \exp (2 \alpha t)))+2 \ln (1-A)=2 \alpha t+2 \ln \left(\frac{1-A}{1-A \exp (2 \alpha t)}\right) .
$$

The assumption that $\rho$ does not blow up implies $A \exp (2 \alpha t)<1$, and so there is a positive contribution from the logarithmic term and hence

$$
\int_{0}^{t} \nabla \cdot f d s>2 \alpha t
$$

Let us rewrite (20) using (16), thus we obtain

$$
\operatorname{det}(\Phi)=\frac{\alpha^{2}}{\left(\rho_{0}^{2}-\left(\rho_{0}^{2}-\alpha\right) \exp (2 \alpha t)\right)^{2}} \exp (2 \alpha t)
$$

which is the same functional expression as (14).

Now suppose that a total time $T$ is taken from $\left(x_{0}, y_{0}\right)$ to $\left(x_{0}^{\prime}, y_{0}^{\prime}\right)$, with a passage of time $t_{0}+t_{1}$ from $\left(x_{0}, y_{0}\right)$ to $\left(x_{2}, y_{2}\right)$ and then $N_{1}-1$ segments each for the time $t_{i}=n_{i} \tau$ (with $n_{i}$ being a positive integer depending on the index $i$ of the short segment) using (6) in the zig-zag part. Then the determinant along the flow from $D$ back to $D$, say $\operatorname{det}(\Phi)_{T}$, is the product

$$
\operatorname{det}(\Phi)_{T}=\frac{\alpha^{2} \exp \left(2 \alpha t_{0}+t_{1}\right)}{\left(\rho_{0}^{2}-\left(\rho_{0}^{2}-\alpha\right) \exp \left(2 \alpha t_{0}+t_{1}\right)\right)^{2}} \Pi_{i=2 k+1}^{2 N_{1}-1} \frac{\alpha^{2} \exp \left(2 \alpha t_{i}\right)}{\left(\rho_{i}^{2}-\left(\rho^{2}-\alpha\right) \exp \left(2 \alpha t_{i}\right)\right)^{2}}
$$

where $k=1,2,3, \cdots$. Therefore, we obtained precisely the same functional expression for the full determinant as in the previous case (cf equation (15)). 
Using (15) we calculated the determinant $\operatorname{det}(\Phi)_{T}$ of system (6) using parameter values as set in Sec. 3, with $\tau=0.001$. Setting the initial conditions to $x_{0}=$ $[1.0999 ; 0.01542]$ we find $\operatorname{det}(\Phi)_{T}=4.1016$. Variations of the initial conditions in the neighborhood of the grazing point $\mathbf{x}_{\mathbf{g}}=[1.1 ; 0.01542]$, implies variations of $\operatorname{det}(\Phi)_{T}$. For instance for $x_{0}=[1.0995 ; 0]$ we find $\operatorname{det}(\Phi)_{T}=4.1052$, and for $x_{0}=[1.0999 ; 0.001]$ $\operatorname{det}(\Phi)_{T}=4.1158$. Clearly, variations in the initial conditions of the order even greater than $\tau=0.001$ do not significantly change the determinant and hence the existence of positive Lyapunov exponent is confirmed by our simple numerical experiment.

\section{Generalisation: planar case}

\subsection{Boundedness}

In this section we will consider general conditions on (1) defined by vector fields $F_{i}: \mathbb{R}^{2} \rightarrow \mathbb{R}^{2}$ (we suppress parameter dependance for the clarity of notation), $i=1,2$, with switching surface

$$
H(x)=x_{g}-x
$$

for some constant $x_{g}$, which ensure that the digitally sampled system (6) has bounded solutions. So $F_{1}$ applies if $x<x_{g}$ and $F_{2}$ applies if $x>x_{g}$; a more general switching surface can be considered by change of variable. We are interested in the existence of a bounded region for sufficiently small $\tau>0$. We begin by making a number of assumptions to specify the properties of the system we require, with the notation that $\pi_{x}$ is the projection onto the $x$-coordinate and $\pi_{y}$ the projection onto the $y$-coordinate, so $\pi_{x}(X, Y)=X$ and $\pi_{y}(X, Y)=Y$. As usual $\phi_{i}(x, y, t)$ denotes the solution at time $t$ of the differential equation with vector field $F_{i}$ and initial conditions $(x, y)$.

(A1) There exist $M^{\prime}, N^{\prime}>0$ such that if $-M^{\prime}<y<N^{\prime}$ then

$$
\pi_{x} F_{1}\left(x_{g}, y\right)\left\{\begin{array}{l}
>0 \text { if } y<0 \\
=0 \text { if } y=0 \\
<0 \text { if } y>0
\end{array}\right.
$$

and

$$
\pi_{x} F_{2}\left(x_{g}, y\right)<0, \quad \pi_{y} F_{2}\left(x_{g}, y\right)>0, \quad \text { and } \quad \pi_{y} F_{1}\left(x_{g}, y\right)>0
$$


This has the immediate consequence (by continuity) that for all $\epsilon>0$ sufficiently small, there exist strictly positive numbers $M$ and $N, m_{i}$ and $n_{i}(i=0,1,2)$ depending on $\epsilon$ such that for all points in the closed strip

$$
L_{\epsilon}=\left\{(x, y)|-M \leq y \leq N,| x-x_{g} \mid<\epsilon\right\}
$$

we have

$$
-m_{0}<\pi_{x} F_{1}(x, y)<m_{2}, \quad n_{0}<\pi_{y} F_{1}(x, y)<n_{1}
$$

and

$$
m_{1}<-\pi_{x} F_{2}(x, y)<m_{2}, \quad n_{3}<\pi_{y} F_{2}(x, y)<n_{2}
$$

Moreover,

$$
\pi_{x} F_{1}(x, N)<0, \quad\left|x-x_{g}\right|<\epsilon
$$

(A2) There exists $T>0$ such that

$$
\pi_{x} \phi_{1}\left(x_{g}, 0, T\right)=x_{g}, \quad \pi_{y} \phi_{1}\left(x_{g}, 0, T\right)<0
$$

with $\pi_{x} \phi_{1}\left(x_{g}, 0, t\right)<x_{g}$ for $0<t<T$. Moreover, for all $\epsilon>0$ sufficiently small, there exist $\eta_{1}$ and $\eta_{2}$ such that $\pi_{x} \phi_{1}\left(x_{g}, 0, \eta_{1}\right)=\pi_{x} \phi_{1}\left(x_{g}, 0, T-\eta_{2}\right)=x_{g}-\epsilon$ and $\pi_{x} \phi_{1}\left(x_{g}, 0, t\right)<x_{g}-\epsilon$ for the non-empty set $\eta_{1}<t<T-\eta_{2}$.

Note that assumptions (A1) and (A2) imply that there is a sliding orbit for the switched system (1) consisting of $\phi_{1}\left(x_{g}, 0, t\right), 0 \leq t<T$ and the passage along $x=x_{g}$ from $\phi_{1}\left(x_{g}, 0, T\right)$ back to $\left(x_{g}, 0\right)$.

Once again continuity has some simple consequences: there exists $\epsilon_{c}>0$ such that for all $\epsilon \in\left(0, \epsilon_{c}\right]$ and all $y$ in a sufficiently small neighbourhood $V_{\epsilon}$ of zero, if $\left|\epsilon_{i}\right| \leq \epsilon$, $i=1,2$, then there exist positive functions $T_{0}\left(y, \epsilon_{1}, \epsilon_{2}\right)$ taking values close to $T$ such that

$$
\pi_{x} \phi_{1}\left(x_{g}+\epsilon_{1}, y, T_{0}\right)=x_{g}+\epsilon_{2} .
$$

The specification that $T_{0}$ is close to $T$ is a somewhat informal way of specifying that the solution $\phi_{1}\left(x_{g}+\epsilon_{i}, y, t\right)$ with $0 \leq t \leq T_{0}$ leaves a strip close to $x=x_{g}$ and then re-enters it having gone once round a loop close to the orbit of (A2).

Combining this remark with the consequences of (A1) we see that there is $\epsilon_{0}>0$ such that $L_{\epsilon_{0}}$ can be chosen, reducing $N$ if necessary, so that

$$
L_{\epsilon_{0}} \cap\{y \geq 0\} \subset V_{\epsilon_{0}},
$$


i.e. all trajectories started in $L_{\epsilon_{0}}$ close to $\left(x_{g}, 0\right)$ with $y \geq 0$ eventually return to $L_{\epsilon_{0}}$ below $y=0$. Clearly, if $0<\epsilon<\epsilon_{0}$ then $L_{\epsilon}$ will also satisfy (31).

Our final assumption ensures that the surface with $\pi_{x} F_{1}(x, y)=0$ is well behaved near $\left(x_{g}, 0\right)$.

(A3) The partial derivative of $\pi_{x} F_{1}(x, y)$ with respect to $y$ is non-vanishing at $\left(x_{g}, 0\right)$ :

$$
\frac{\partial\left(\pi_{x} F_{1}\right)}{\partial y}\left(x_{g}, 0\right)=A \neq 0
$$

An immediate consequence of the Implicit Function Theorem is that if

$$
B=\frac{\partial\left(\pi_{x} F_{1}\right)}{\partial x}\left(x_{g}, 0\right)
$$

then there is a unique continuously differentiable local solution $y=g(x)$ to the equation $\pi_{x} F_{1}(x, y)=0$ with $g\left(x_{g}\right)=0$, and

$$
y \sim-\frac{B}{A}\left(x-x_{g}\right) .
$$

The geometry established by the arguments above is shown in Figure 7, which is enough to prove bounded motion close to the sliding orbit in the digitally sampled system of our interest for all sufficiently small $\tau>0$. The region is constructed as follows.

For $\epsilon>0$ sufficiently small, and $\tau>0$ sufficiently small let

$$
g_{1}=y_{m}, \quad g_{2}=g\left(x_{g}-\epsilon\right)
$$

where $y_{m}$ is defined by the right hand side of (34), and define $\mathcal{D}$ to be the annular region bounded by the curves (in the notation of the discussion above, in particular $(30))$

$$
\begin{aligned}
& \phi_{1}\left(x_{g}+\epsilon, g_{1}, t\right), \quad 0 \leq t \leq T_{0}\left(g_{1}, \epsilon, \epsilon\right), \\
& \phi_{1}\left(x_{g}-\epsilon, g_{2}, t\right), \quad 0 \leq t \leq T_{0}\left(g_{2},-\epsilon,-\epsilon\right), \\
& x=x_{g}+\epsilon, \quad \pi_{y} \phi_{1}\left(x_{g}+\epsilon, g_{1}, T_{0}\left(g_{1}, \epsilon, \epsilon\right)\right) \leq y \leq g_{1}, \\
& x=x_{g}-\epsilon, \quad \pi_{y} \phi_{1}\left(x_{g}-\epsilon, g_{2}, T_{0}\left(g_{2},-\epsilon,-\epsilon\right)\right) \leq y \leq g_{2} .
\end{aligned}
$$




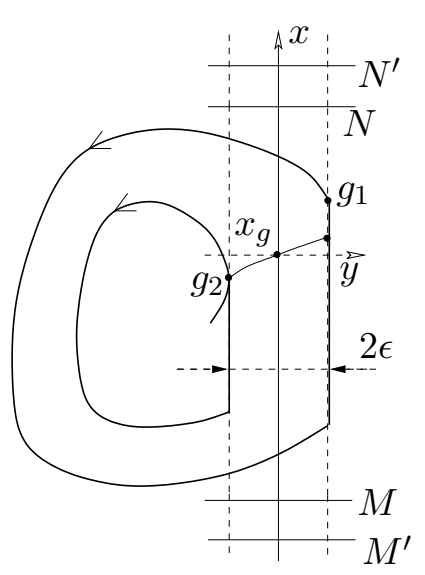

Fig. 7. Bounded annular region in general planar Filippov type systems with digital sampling

Proposition 1 Suppose that a digitally sampled system (6) satisfies (A1)-(A3). Then there exist $\epsilon>0$ such that for all $\tau>0$ sufficiently small, if a solution starts in $\mathcal{D}$ with $x<x_{g}-\epsilon$, then it will stay in $\mathcal{D}$ for all future times.

Proof: Fix $\epsilon_{0}$ so that $L_{\epsilon_{0}}$ satisfies (31) and the constants of (26) and (27) are fixed. For $\epsilon<\epsilon_{0}$, consider a solution started in $L_{\epsilon}$ with $y<0$ and $x_{g}-\epsilon<x<x_{g}$. After time $\tau$ (A1) implies using (26) that $y<n_{1} \tau$. If the solution is then in $x>x_{g}$, it will have returned to $x<x_{g}$ after at most a further $\left\lceil\epsilon /\left(m_{1} \tau\right)\right\rceil \tau$-time steps by (27), i.e. it will have

$$
y<y_{m}=n_{1} \tau+n_{2}\left\lceil\frac{\epsilon}{m_{1} \tau}\right\rceil \tau .
$$

Choose $\epsilon$ such that for all $\tau>0$ sufficiently small the points $\left(x, y_{m}\right)$ with $\left|x-x_{g}\right|<\epsilon$ are in $L_{\epsilon_{0}}$ and

$$
m_{2} \tau<\epsilon
$$

with $m_{2}$ defined in (26) and (27).

If a solution starts in $\mathcal{D}$ with $x<x_{g}-\epsilon$ then it will move around the annulus until it enters $x>x_{g}$ and starts moving up inside the cylinder $\left|x-x_{g}\right|<\epsilon$. Note that by (35) it overshoots $x=x_{g}$ by at most $\epsilon$ in either direction.

Now suppose that at time $n \tau$ a solution is at $\left(x_{0}, y_{0}\right)$ inside the annular region $\mathcal{D}$ with $y_{0}<0$ and $\left|x-x_{g}\right|<\epsilon$. Then since the defining equations are time independent, we may reset time to $t=0$.

If $x_{0} \geq x_{g}$ and the flow is defined by $F_{2}$, it will be upwards and to the left. Let $m_{1} \tau$ be the first time $\phi_{2}\left(x_{0}, y_{0}, k \tau\right)$ lies in $x<x_{g}$ for integer $k>0$. By (35) $\pi_{x} \phi_{2}\left(x_{0}, y_{0}, m_{1} \tau\right)>$ $x_{g}-\epsilon$, and the solution curve $\phi_{2}\left(x_{0}, y_{0}, t\right), 0<t<k \tau$, certainly enters $x<x_{g}$ after 
no greater than $\left\lceil\epsilon / m_{1} \tau\right\rceil \tau$-time steps and hence has $y<n_{2}\left\lceil\epsilon / m_{1} \tau\right\rceil \tau$, cf (34). Thus it lies below the upper boundary of $\mathcal{D}$. Thus either $\phi_{2}\left(x_{0}, y_{0}, k \tau\right)$ is in $\mathcal{D}$ with $x<x_{g}$ and $y<0$, or it is in $\mathcal{D}$ with $x<x_{g}$ and $y \geq 0$. In both cases the next part of the flow is under $F_{1}$.

Let us focus first on the latter case and consider a trajectory starting in $\mathcal{D}$ with $x_{g}-\epsilon<$ $x<x_{g}$ and $y \geq 0$ but below $\phi_{1}\left(x_{g}+\epsilon, g_{1}, t\right)$. The solution is thus trapped either between $\phi_{1}\left(x_{g}-\epsilon, g_{2}, t\right)$ and $\phi_{1}\left(x_{g}, 0, t\right)$ or $\phi_{1}\left(x_{g}, 0, t\right)$ and $\phi_{1}\left(x_{g}+\epsilon, g_{1}, t\right)$ (together, possibly, with part of the line $x=x_{g}-\epsilon$ in $x>0$ which trajectories cross in the direction of decreasing $x$ ) until such time as it re-enters $x>x_{g}$ with $y<g(x)$ and $x<x_{g}+\epsilon$ by (35) again. For the whole of this passage it is, by definition, in $\mathcal{D}$ and hence at the end of this passage (an integer multiple of $\tau$ later), we have $x \geq x_{g}$, $y<0$, and the flow defined by $F_{2}$ again.

Now suppose the solution starts at $\left(x_{0}, y_{0}\right)$ in $x \leq x_{g}$ with $y<0$. Let $n$ be the smallest positive integer such that either $\phi_{1}\left(x_{0}, y_{0}, n \tau,\right)$ has $x \leq x_{g}-\epsilon$ or $x>x_{g}$ (note that $n$ exists by the geometry of $\mathcal{D}$ ). There are three cases.

If $\phi_{1}\left(x_{0}, y_{0}, n \tau,\right)$ has $x>x_{g}$ and $y<0$ then since $x<x_{g}+\epsilon$ by (35) and $y>y_{0}$ it lies in $\mathcal{D}$. Hence after relabelling the origin of time it is a case discussed earlier.

If $\left(x^{\prime}, y^{\prime}\right)=\phi_{1}\left(x_{0}, y_{0}, n \tau\right)$ has $x^{\prime}>x_{g}$ and $y^{\prime}>0$ then note that $\phi_{1}\left(x_{0}, y_{0},(n-1) \tau\right)$ still has $x \leq x_{g}$, as if this were not the case, the trajectory would leave the strip without entering $x>x_{g}$. Hence $y^{\prime}<n_{1} \tau$ and so by (34) the next portion of trajectory under

$F_{1}$ will lie below $\phi_{1}\left(x_{g}+\epsilon, g_{1}, t\right)$ with $x_{g}-\epsilon \leq x \leq x_{g}$, and this has already been discussed.

The final possibility is that $\phi_{1}\left(x_{0}, y_{0}, n \tau\right.$, ) has $x \leq x_{g}-\epsilon$, in which case it lies between $\phi_{1}\left(x_{g}+\epsilon, g_{1}, t\right)$ and $\phi_{1}\left(x_{g}-\epsilon, g_{2}, t\right)$ and the argument already made ensures that the solution remains in $\mathcal{D}$ and eventually has $x>x_{g}$ with $y<0$ at an integer multiple of $\tau$.

\subsection{The estimate of the time of evolution along the switching line $\Sigma$}

In the following section we will estimate the times of evolution of the zig-zag part of the trajectory along the switching line $\Sigma$ assuming that the discretization time $\tau$ is sufficiently small. Thus, we will approximate $F_{1}$ and $F_{2}$ using constant vector fields for $\mathbf{x}$ living in some sufficiently small neighborhood of a point on $\Sigma$. Using two- 
segments zig-zag part of the trajectory, one segment generated by $F_{1}$ and the second one by $F_{2}$ we will obtain a vector field, say $\bar{F}$, that we will use to approximate the evolution of the system ignoring the presence of switchings. Let us first denote the constant approximations of $F_{1}$ and $F_{2}$ by $\bar{F}_{1}$ and $\bar{F}_{2}$ respectively. Let

$$
\bar{F}_{1}=\left(\begin{array}{l}
a_{1} \\
b_{1}
\end{array}\right), \quad \bar{F}_{2}=\left(\begin{array}{l}
a_{2} \\
b_{2}
\end{array}\right),
$$

where $a_{1}, b_{1}, a_{2}$ and $b_{2}$ are constants depending on $\mathbf{x}$. In our case, on $\Sigma$, within the region of interest $a_{1}>0$, and $a_{2}<0$.

Let $t_{1}=n \tau, t_{2}=m \tau$ be the times of evolution of a segment generated by $\bar{F}_{1}$ and $\bar{F}_{2}$ respectively, and $n, m$ be positive integers. Then the approximate position vector that gives the position of a trajectory ignoring the presence of a switch can be given by $\bar{s}=\bar{F}_{1} t_{1}+\bar{F}_{2} t_{2}$, and the velocity vector corresponding to $\bar{s}$, say $\bar{F}$, is then given by

$$
\bar{F}=\frac{\bar{s}}{t_{1}+t_{2}}=\bar{F}_{1} \frac{t_{1}}{t_{1}+t_{2}}+\bar{F}_{2} \frac{t_{2}}{t_{1}+t_{2}} .
$$

Given $\alpha=\frac{t_{1}}{t_{1}+t_{2}}$ we can write $\bar{F}=\bar{F}_{1} \alpha+\bar{F}_{2}(1-\alpha)$. Let $\left(x_{0}, y_{0}\right)$ be the initial point of the two-segment zig-zag trajectory which we are considering. Define $\left(x_{0}^{L}, y_{0}^{L}\right)$ as a point sufficiently close to $\left(x_{0}, y_{0}\right)$ such that $x_{0}^{\Sigma}=a_{1} n \tau+x_{0}^{L} \in \Sigma$, and $\left(x_{0}^{U}, y_{0}^{U}\right)$ as a point sufficiently close to $\left(x_{0}, y_{0}\right)$ such that $\tilde{x}_{0}^{\Sigma}=a_{1}(n-1) \tau+x_{0}^{U} \in \Sigma$. Thus any point in a sufficiently small neighborhood of $\left(x_{0}, y_{0}\right)$ with $x_{0} \in\left(x_{0}^{L}, x_{0}^{U}\right)$ crosses $\Sigma$ within time $\tau$. Let us assume without loss of generality that $\left|a_{1}\right|>\left|a_{2}\right|$ (we will treat the case $\left|a_{2}\right|>\left|a_{1}\right|$ later, in the equivalent fashion). This implies that $n=1$ since the furthest distance from the switching surface along the $x$-axis which can be reached be vector field $\bar{F}_{2}$ has the magnitude $\left|a_{2}\right| \tau$ and this distance is covered by a trajectory generated by $\bar{F}_{1}$ within time $\tau$ (considering sufficiently small neighborhood of $\left.\left(x_{0}, y_{0}\right)\right)$. Therefore, we can assume without loss of generality that $t_{1}=\tau, t_{2}=m \tau$, and hence

$$
\alpha=\frac{\tau}{\tau+m \tau} .
$$

We now need to estimate $m$. Note that the distance covered by the trajectory following $\phi_{1}$ within time $\tau$ is $\left|a_{1}\right| \tau$ which is approximately equal to the distance covered by the trajectory following $F_{2}$ in $m$ steps. Therefore, we can write

$$
\left|a_{1}\right| \tau+\Delta x=m \tau\left|a_{2}\right|
$$

where $\Delta x \in\left[0, \tau\left|a_{2}\right|\right]$, and $m=\left[\frac{\left|a_{1}\right|}{\left|a_{2}\right|}\right]$. In view of (37) we can approximate the integer 
$m$ in (36) by a number $\left|a_{1}\right| /\left|a_{2}\right|$. This is justifiable for sufficiently small $\tau$ since then $\Delta x$ is small. This gives

$$
\hat{\alpha}=\frac{1}{1+\left|a_{1}\right| /\left|a_{2}\right|},
$$

and the vector field $\hat{F}$ which approximates $\bar{F}$ can be given as

$$
\hat{F}=\hat{\alpha} \bar{F}_{1}+(1-\hat{\alpha}) \bar{F}_{2} .
$$

Note that in the current case the point furthest to the left of $\Sigma$ is $\left|a_{2}\right| \tau$ distance away from $\Sigma$.

Let us now consider the case when $\left|a_{2}\right|>\left|a_{1}\right|$. In this case $m=1$ and

$$
n\left|a_{1}\right| \tau=\tau\left|a_{2}\right|+\Delta x
$$

with $\Delta x \in\left[0, \tau\left|a_{1}\right|\right], n=\left\lceil\frac{\left|a_{2}\right|}{\left|a_{1}\right|}\right\rceil$. Again, approximating $n$ by $\hat{n}=\left|a_{2}\right| /\left|a_{1}\right|$ gives

$$
\hat{\alpha}=\frac{\left|a_{2}\right| /\left|a_{1}\right| \tau}{\left|a_{2}\right| /\left|a_{1}\right| \tau+\tau}=\frac{1}{1+\left|a_{1}\right| /\left|a_{2}\right|}
$$

which is the same expression as (38). Note that in either case for $\tau \rightarrow 0 \Delta x \rightarrow 0$ and the error of our approximation tends to 0. Moreover the relative error between $\alpha$ and $\hat{\alpha}$ is small provided that $a_{1}$ and $a_{2}$ are of different orders.

Let us now compare expression (38) with an expression for $\alpha$ obtained in the case when $\tau=0$ and the system follows sliding motion. In that case the sliding vector field is given by (3) and the parameter corresponding to $\alpha$ is $\alpha_{s} \in[0,1]$, and $\alpha_{s}=\frac{\left\langle H_{x} F_{2}\right\rangle}{\left\langle H_{x}\left(F_{2}-F_{1}\right)\right\rangle}$, where $H_{x}$ is the vector normal to $\Sigma$. Clearly in our case $\alpha_{s}=\frac{a_{2}}{a_{2}-a_{1}}=\frac{1}{1-a_{1} / a_{2}}$. Throughout sliding region, where $F_{s}$ applies, $a_{1}>0$ and $a_{2}<0$, thus we obtain $\alpha_{s}=\frac{1}{1+\left|a_{1}\right| /\left|a_{2}\right|}$ which is the same expression as (38). Therefore, the sliding vector field is a natural approximation of the zig-zag evolution along $\Sigma$ and in the limit $\tau \rightarrow 0$ both vector field $F_{s}$ and $\hat{F}$ coincide.

We can then approximate the time of evolution following the zig-zag paths along $\Sigma$ ignoring the switching by the integral

$$
T^{S}=\int_{y_{1}}^{y_{2}}\langle\bar{F} \mathrm{~d} s\rangle
$$


where $\langle$,$\rangle denotes the dot product, \mathrm{d} s$ is a vector along $\Sigma$, and the integral is parameterized by the $y$ component of the state vector $\mathbf{x}$. The boundaries of integration are values of the $y$ component of the state vector at the first crossing with $\Sigma$, when the zig-zag trajectory starts, and the last crossing with $\Sigma$ when the zig-zag trajectory ends.

We can further approximate the time of evolution along the zig-zag part of the trajectory spend by following vector field $F_{2}$ using (38). If $\left|a_{1}\right|$ is in average twice as large as $\left|a_{2}\right|$ along $\Sigma$ then $\hat{\alpha}=1 / 3$. This implies further that $2 / 3$ of the time $T^{s}$ is generated by vector field $F_{2}$. Thus by considering different relations between $a_{1}$ and $a_{2}$ we can estimate the maximum time of evolution along the zig-zag part of the trajectory generated by $F_{2}$. Let us call by $R F$ the coefficient that describes the average ratio of $\left|a_{1}\right| /\left|a_{2}\right|$ for $x \in \Sigma$. Therefore, the time spend evolving along $\Sigma$ using vector field $F_{2}$ can be approximated by

$$
t_{F_{2}}=R F \int_{y_{1}}^{y_{2}}\langle\bar{F} \mathrm{~d} s\rangle
$$

where $R F \in(0,1)$. Using (16) we can then determine, in the case of general vector fields $F_{1}$ and $F_{2}$, if the determinant along the two flows can be greater than one and hence determine if a general switched planar system is characterized by a positive Lyapunov exponent. Namely, assuming that vector field $F_{1}$ is characterized by a positive expansion rate, the amount of contraction that can be introduced into the system that allows to preserve the existence of chaotic behavior depends on the time of evolution using contracting flow which would be flow $\phi_{2}$. The contraction is measured by the determinant

$$
\exp \left(\int_{0}^{t_{F_{2}}} \nabla \cdot F_{2} d t\right)
$$

Therefore, if the product

$$
\exp \left(\int_{0}^{t} \nabla \cdot F_{1} d t\right) \exp \left(\int_{0}^{t_{F_{2}}} \nabla \cdot F_{2} d t\right)
$$

is greater than 1 the existence of a positive Lyapunov exponent along the trajectory is guaranteed ( $t$ is the average time of evolution using the expanding vector field under the recurring motion). 


\section{$5 \quad$ Higher-dimensional systems}

We believe that the behaviour observed in our two dimensional example is typical not only for planar systems, but also for $n$-dimensional Filippov type systems with sliding motion and digital sampling. In this section we will present numerical investigations of a third order-relay feedback system without and with the digital sampling. We will describe numerical findings for two cases. In the first case the self-sustained periodic oscillations are preserved under the introduction of the digital sampling and in the second case the introduction of the digital sampling induces chaotic oscillations. Detailed analysis of this system will be presented elsewhere. Here, we only wish to stress that the mechanism leading to the onset of chaos from periodic orbits due to an introduction of digitization, uncovered for planar systems, is also observed in higherdimensions and applies to a wide range of systems in relevance to applications. The relay feedback system under investigation that we consider has the following state space representation

$$
\dot{x}=A x+B u, \quad y=C x, \quad u=-\operatorname{sgn} y,
$$

where

$$
A=\left(\begin{array}{ccc}
-(2 \zeta \omega+\lambda) & 1 & 0 \\
-\left(2 \zeta \omega \lambda+\omega^{2}\right) & 0 & 1 \\
-\lambda \omega^{2} & 0 & 0
\end{array}\right), \quad B=\left(\begin{array}{c}
k \\
2 k \sigma \rho \\
k \rho^{2}
\end{array}\right), \quad x=\left(\begin{array}{l}
x_{1} \\
x_{2} \\
x_{2}
\end{array}\right), \quad C=\left(\begin{array}{c}
1 \\
0 \\
0
\end{array}\right)^{T} .
$$

The switching surface is given by $\left\{y=C x=x_{1}=0\right\}$ and vector field $F_{1}=A x-B$, and $F_{2}=A x+B$. System (40) is given in so-called observer canonical form [14]. The dynamics of (40) has been extensively studied in [15]. There, stable self-sustained oscillations as well as chaotic dynamics have been observed.

We will modify (40) by introducing the digital sampling of the control variable $y$ similarly to the planar case. Namely the switchings between vector fields $F_{1}$ and $F_{2}$ will occur at $n \tau$ time instances when $y(n \tau)$ changes its sign ( $n$ is a positive integer). Let us consider the following parameters: $\zeta=0.1, \omega=1, k=1, \sigma=-1$, and $\rho=0.4$. As depicted in Fig. 8(a) at these parameter values the system exhibits symmetric limit cycles with a segment of sliding motion (see Fig. 9(a) for a zoom into the region along the switching surface $\Sigma$ where sliding takes place). We will then introduce the sampling time $\tau$ to the output variable $y$. Introduction of the positive sampling, in this case, does not appear to lead to the onset of chaotic dynamics. As 


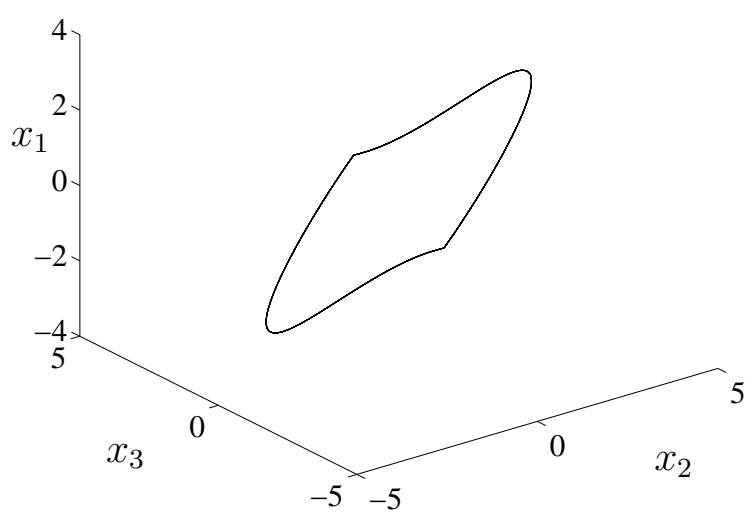

(a)

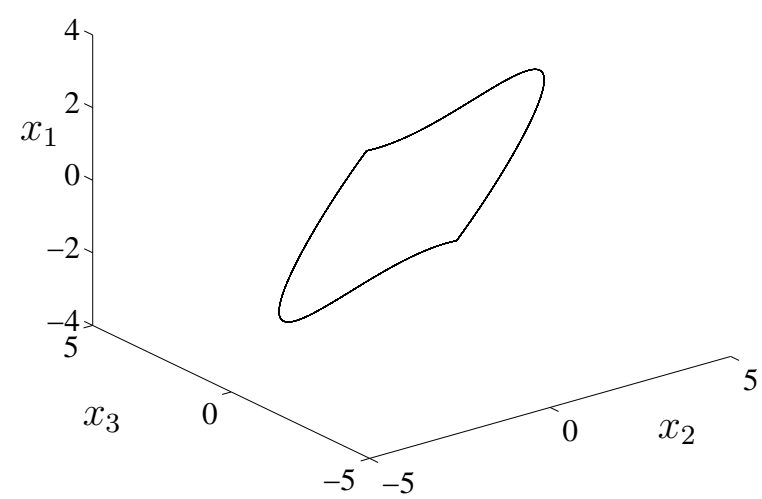

(b)

Fig. 8. Periodic orbit of system (40) (a) with a segment of sliding when no digital sampling is applied to the system, and (b) with digital sampling $\tau=0.005$. Other parameters are set to $\zeta=0.1, \omega=1, k=1, \sigma=-1$, and $\rho=0.4$.

depicted in Fig. 8(b), for $\tau=0.005$, a periodic orbit is preserved with the difference being the destruction of the sliding segment. Instead of sliding the system exhibits a switching along $\Sigma$ (compare figures Fig. 9 (a) and (b)).

Let us now decrease $\zeta$, and $\rho$ in system (40), when no digital sampling is applied. As depicted in Fig. 10(a) for $\zeta=-0.07, \omega=1, k=1, \sigma=-1$, and $\rho=0.05$ the system still exhibits stable periodic oscillations with sliding. Let us now introduce digital sampling. This time we observe apparent thickening of the attractor. The observed attractor is a chaotic attractor. In Fig. 10(b) we depict the attractor born under after we increased $\tau$ to $\tau=0.005$. Notice that the eigenvalues of the characteristic equation of matrix $A$ are $\mu_{1,2}=-\zeta \omega \pm \sqrt{\zeta^{2}-1}, \mu_{3}=-\lambda$. Therefore, for $\zeta$ negative we observe an expansion of a volume of phase space along the flow. This will lead to the existence of a positive Lyapunov exponent along the attractor which can be shown using the method presented in Sec. 3.4 and by considering the cartesian setting.

A more detailed description of the multi-dimensional case will be presented elsewhere, but the important message from this example is that if the $F_{1}$ component of a stable sliding orbit is sufficiently unstable then we appear to observe the same effect as was detailed for the planar case earlier. 


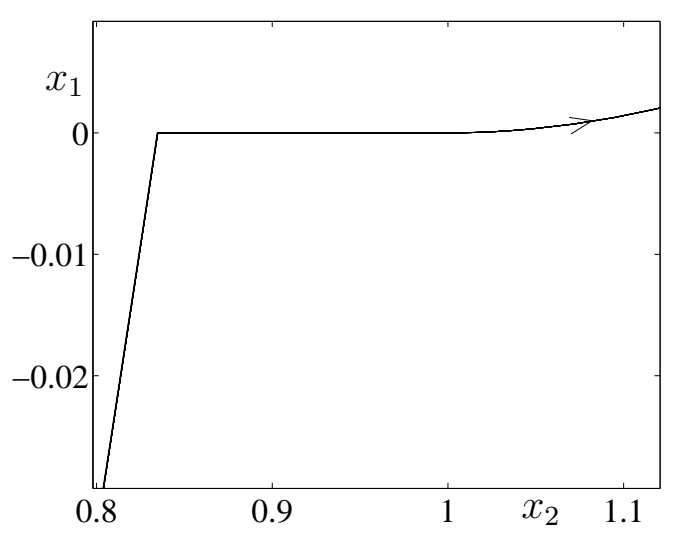

(a)

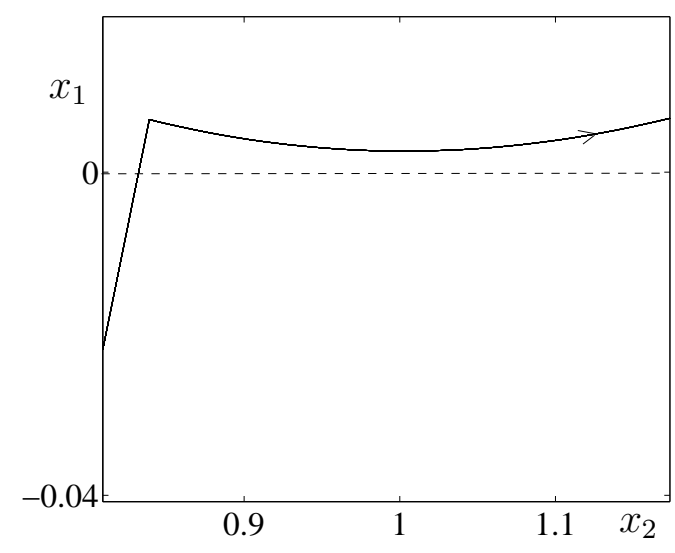

(b)

Fig. 9. Zoom into the segment of periodic orbit from figure 8 along the switching line when no sampling is applied (a), and with digital sampling $\tau=0.005$ (b). Note that the sliding part of the trajectory is destroyed.

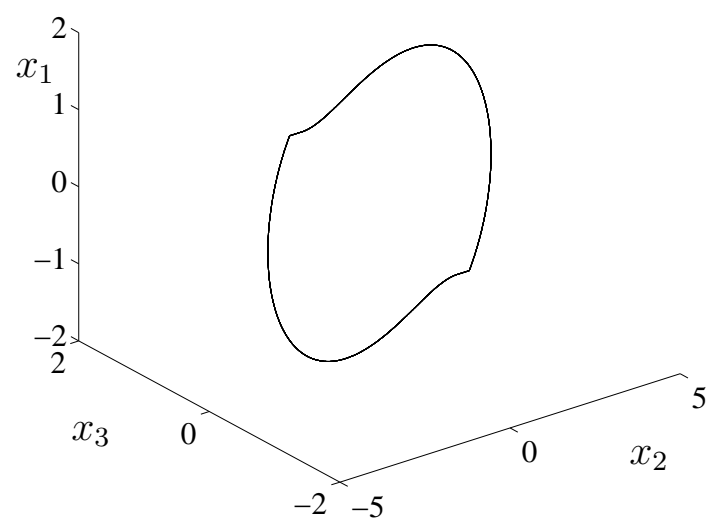

(a)

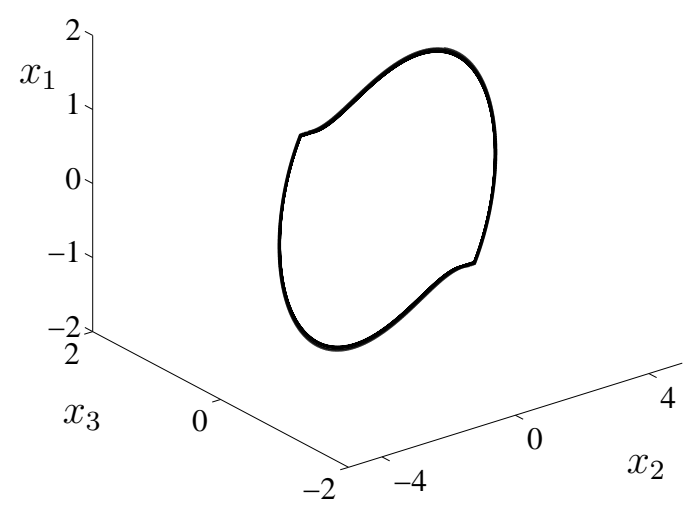

(b)

Fig. 10. Periodic orbit of system (40) (a) with a segment of sliding when no digital sampling is applied to the system, and (b) with digital sampling $\tau=0.005$. Other parameters are set to $\zeta=-0.07, \omega=1, k=1, \sigma=-1$, and $\rho=0.05$.

\section{Conclusions}

In the paper we study Filippov type systems with digital sampling. It is shown that digital sampling may lead to the onset of chaotic dynamics. A simple example is studied in detail to reveal the mechanism leading to chaotic dynamics. The existence of at least one chaotic attractor is proved rigorously, but we have not excluded the possibility that other attractors exist. Thus uniqueness (or topological transitivity) 
of the attractor in the bounded region is still an open problem. We conjecture that the chaotic attractor is indeed unique.

It is shown that the size of the chaotic attractor, measured as the distance between the chaotic attractor and a stable cycle of continuously sampled orbit is linear for sufficiently small values of the sampling time $\tau$. The results are generalized to planar Filippov type systems with digital sampling. Using planar systems we also show that in the limit when the sampling time $\tau \rightarrow 0$, the Filippov's method that gives the sliding flow of continuously sampled system, converges to the vector field of the discretely sampled system. This result can then be used to approximate the time of evolution along the switching surface which in turn can be utilized to determine if the expansion/contruction due to the zig-zag evolution along the switching surface can qualitatively alter system dynamics.

Based on our finding we believe that analyzed dynamics will also occur in $n$-dimensional Filippov type systems when the variable that determines switching between different vector fields is sampled at discrete time intervals. To justify this conjecture we investigate a third order relay feedback system and introduce digital sampling to continuously sampled control variable. It turns out that, indeed, in certain instances we observe a transition from a stable orbit with a segment of sliding motion, existing in a continuously sampled system, to a chaotic attractor of digitally sampled system. Two scenarios are considered. In the first case the introduction of digital sampling destroys the sliding segment - instead of the sliding flow the system switches along the switching surface until it leaves off the switching plane. In the second case the introduction of digital sampling leads to the creation of chaotic dynamics.

It was shown in [16] that discrete control typically creates a chaotic attractor in the vicinity of unstable equilibrium. There are certain similarities that lead to the onset of chaos in our case and in the case investigated in [16]. In our case we deal with periodic orbits and if we wish to stabilize an unstable orbit that might correspond to some desired oscillatory behavior of a control system the application of the digital control may quite likely lead to the creation of chaotic orbit whereas control provided in continuous time will lead to a stable orbit with a segment of sliding. In [16] it is claimed that artificial neural networks with reinforcement learning are known to be able to learn such a control scheme. It would be interesting to investigate further the link between our findings and neural networks with reinforcement learning. We should also mention that the onset of chaotic dynamics triggered by this mechanism is similar to an abrupt transition from a stable periodic orbit with sliding to a small scale chaotic dynamics that might occur in Filippov type systems under an introduction of an arbitrarily small time delay in the switching function [17]. 


\section{Acknowledgments}

Research partially funded by EPSRC grant EP/E050441/1 and the University of Manchester.

\section{References}

[1] C. B. Kuo. Digital Control Systems. Saunders College Publishing, Fort Worth, London, 1992 .

[2] C. A. Rabbath and N. Hori. A framework for discrete-time models of continuous-time systems. In Proceedings of the American Control Conference, pp. 2578-2583, Arlington 2001.

[3] G. Haller and G. Stépán. Micro-chaos in digital control. Journal of Nonlinear Science, 6:415-448, 1996.

[4] E. Enikov and G. Stépán. Micro-chaotic motion of digitally controlled machines. Journal of Vibration and control, 4:427-443, 1998.

[5] G. Stépán, L. E. Kollar and J. S. Hogan. Sampling delay and backlash in balancing systems. Periodica Polytechnica, 44:77-84, 2000.

[6] J. Guldner, V. I. Utkin, and J. Ackermann. Sliding mode control approach to automatic car steering. In Proceedings of the American Control Conference, pp. 1969-1973, 1994.

[7] D. Liberzon. Quantization, time delays, and nonlinear stabilization. IEEE TRANSACTIONS ON AUTOMATIC CONTROL, 51(7):1190-1195, July 2006.

[8] K. Lee and A. H. Haddad. Stabilization of discrete-time quantized control system. In Proceedings of the American Control Conference, pp. 3506-3511, 2002.

[9] J. H. Braslavsky, E. Kofman, and F. Felicioni. Effects of time quantization and noise in level crossing sampling stabilization. In In Proceedings of AADECA, 2006.

[10] X. Xia and G. Chen. On delta-modulated control: A simple system with complex dynamics. Chaos, Solitons and Fractals, 33:1314-1328, 2007.

[11] A. F. Filippov. Differential equations with discontinuous right hand sides. Kluwer Academic Publishers, Dortrecht, 1988.

[12] R. Seydel. From equilibrium to chaos. Practical bifurcation and stability analysis. Springer-Verlag, New York, London, 1994. 
[13] P. Glendinning. Stability, instability and chaos: an introduction to the theory of nonlinear differential equations. Cambridge University Press, 1994.

[14] R. C. Dorf and R. H. Bishop. Modern Control Systems. Prentice-Hall, Upper Saddle River, New Jersey 07458, 2001.

[15] M. di Bernardo, K. H. Johansson, and F. Vasca. Self-oscillations and sliding in relay feedback systems: Symmetry and bifurcations. International Journal of Bifurcations and Chaos, 11(4):1121-1140, 2001.

[16] A. Potapov and M. K. Ali. Chaotic neural control. Physical Review E-Statistical, Nonlinear, and Soft Matter Physics , 63(4):046215, 2001.

[17] J. Sieber. Dynamics of delayed relay systems. Nonlinearity, 19:2489-2527, 2006. 